\title{
Pride and shame in child and family protection: Emotions and the search for humane practice
}

\author{
Matthew Gibson \\ Policy Press, Great Britain, 2019 \\ ISBN: 978-1-4473-4481-0, pp.253, Paperback, \$NZD59
}

$\mathrm{H}$ ave you ever wondered what it's like to work in child and family social work? Have you ever considered what feelings and emotions the social worker and families experience while being part of this journey? As a supervisor of social workers, are you needing to gain a deeper understanding of your supervisee's experience in their chosen fields? As a provider of social services, have you ever considered how the very structure of your service may influence feelings of pride and shame amongst your employers? If your answer is yes to any of these questions, this is the book for you!

Initially, it was the book's title that really caught my eye. Such emotive words as pride and shame stirred my interest. Words that have always resonated with me at a personal level in my search to understand humanity and professionally as a social worker and supervisor in striving to provide and encourage therapeutic supervision that acknowledges the role emotion plays in our practice. As a health social worker of several years, with my major experiences being mental health, child and family protection issues are a smaller part of my practice. As a supervisor, I am now witnessing the impact of this subject via supervisees who share their journeys in child and family social work and so my interest on the subject has been further sparked.

If you are a social worker or a supervisor, the book not only encourages reflection but almost demands it! Thoughts drop into place, align and provide understanding and clarity and, in fact, a wider connection occurs as you realise just how big a role pride and shame play as a culture, within the wider environment of workplaces and indeed as a country which continues to experience the aftermath of the Treaty of Waitangi journey. Its timing could not be more appropriate either, as New Zealand's largest child and family protection agency comes under public scrutiny and we begin to hear the stories of those working within this very organisation.

Matthew Gibson is a social work academic in the Department of Social Policy and Social Work at the University of Birmingham, England. His research interests relate to emotions and professional practice, and particularly, the emotions of pride and shame. Dr Gibson undertook a study on pride and shame in child and family social work and this book is based on his research and is thorough and informative. The book is throroughly referenced, evidenced and well written. I appreciated the visual representations and the case studies included in the book to solidify the meaning of what I was reading.

For me, the book forced me to consider, in more depth, the many different aspects of the issues social workers encounter in this field. As a social worker, I had often experienced the feeling that there was not enough time to adequately do my job and the pressure I sensed that administrative tasks were more important than the therapeutic and practical support I could provide to a family, for example. Gibson speaks of the same concerns after hearing similar stories from the social workers who were interviewed. Furthermore, prioritisation being given to
AOTEAROA NEW ZEALAND SOCIAL WORK 33(4), 89-90. 
written reports to provide to senior staff so that they could be praised for a job well done, for example, and generally having to be engaged in tasks which left them feeling less empathy for the family, less client focused and literally developing an attitude of indifference toward the family-and this is just a snippet of what the book offers!

What a struggle of emotion for the social worker to have to process! It is no wonder feelings of shame occur!

This book further affirmed my belief of the importance of having quality senior management personnel who understand the role that shame and pride play in social work, who support practitoners in decision making and guide them through the many possible potholes that are best avoided during their journey as a developing social worker and throughout their career. The author discusses an example regarding a new service being introduced in an agency. Work was divided and allocated to individuals and the expectation set that individuals would be held to account if the work was not completed and to an expected standard. The team lead/supervisor explains that within a month, they were flooded with referrals the team struggled to meet. The team lead viewed this as a direct result of the new service. Senior management did not and, in fact, viewed it as the team lead "not being on top of it or equal to the task." Feelings of incompetence, shaming of and panic by workers were noted and transferred down the line to workers below. These workers were subjected to a myriad of emotions and a double layer of shame as a result.

The author sums the book's content up well when he speaks about the role self-conscious emotions play in the organisational mahi / work of the interviewed social workers. He speaks of the resistance they experience in responding to organisational expectations by working their way through and regulating their emotions before reacting to the demands and expectations often placed on them. Furthermore, Gibson speaks about the social workers' need to consider whether their reaction is based on past experience/s and fear of further guilt or shame for what they may have done or who they were. Also, how these experiences can make the profession undesirable. It is obvious, the book covers a lot about a juicy and interesting subject!

If you hadn't already pondered the role pride and shame play in understanding this important mahi/work before picking this book up, you will very quickly understand the incredible need for humane practice in this field. If you have been a social worker who has been working hard, do yourself a favour and grab a copy of this book. Pour yourself a pot of tea, gather your favourite snacks, get sorted in your comfy chair and simply begin to read it. I would find it hard to believe you will not be left with a feeling of understanding, empathy, perhaps sadness, enlightenment and an overwhelming desire to get out there and support your fellow social workers with some simple kindness and a deeper understanding. 